\title{
Influence of the Temperature on the Liquid-Liquid-
}

\section{Solid Equilibria of the Water + Ethanol + 1-}

\section{Undecanol Ternary System}

Vicente Gomis*, M. Dolores Saquete, Nuria Boluda-Botella, and Alicia Font.

Chemical Engineering Department, University of Alicante, Apartado 99, E-03080 Alicante, Spain.

KEYWORDS: Experiments; Data; Ethanol; 1- undecanol; Solid-liquid equilibria; Liquid-liquid equilibria; Temperature; Extraction.

\section{ABSTRACT}

Liquid-liquid (L-L), solid-liquid (S-L) and solid-liquid-liquid (S-L-L) equilibrium data for the water - ethanol - 1-undecanol ternary system have been determined experimentally at 275.15 , 278.15, 281.15, 288.15 and $298.15 \mathrm{~K}$ and atmospheric pressure. Different shapes of the equilibrium diagrams have been observed depending on the temperature. A region with three phases (S-L-L) is present in the temperature range between 275.15 and $281.15 \mathrm{~K}$. Above 288.15 $\mathrm{K}$, only a L-L region is observed.

* Corresponding author. E-mail: vgomis@ua.es. Tel.: +34 965903400. Fax: +34 965903826. 


\section{INTRODUCTION}

The ethanol obtained in fermentation of carbohydrates can be separated from the aqueous solution (azeotropic composition) by different methods. Extraction with long-chain alcohols, such as 1-dodecanol [1] and 1-decanol [2], could be a possible way of separation. In these two previous papers, different liquid-liquid, solid-liquid and solid-liquid-liquid equilibria were determined at different temperatures, in order to perform a better separation. In this paper, another straight-chain primary alcohol, 1-undecanol, is utilized in the ethanol-water extraction at different temperatures. This fatty alcohol exists in nature, found naturally in many foods such as fruits (apples, bananas), butter and eggs [3], and could be proposed as an alternative extractant, in environmentally sustainable processes.

In addition, the development of new analytical techniques, such as dispersive liquid-liquid microextraction (DLLME) invented in 2006, requires knowledge of the extraction process, since the drawbacks of this technique are the requirements related to the extraction and disperser solvent [4]. A modification of this technique together with the solidification of floating organic drop (DLLME-SFO) was proposed by Mirzaei et al. [5] for the extraction of heavy metal ions from industrial effluent samples. Among other compounds, they used ethanol as the disperser solvent and 1-undecanol as the extraction solvent. This technique utilizes extraction solvents with lower toxicity than that usually employed in DLLME and faster than other procedures [5].

As a consequence of all these applications, the study of the water + ethanol +1 -undecanol equilibrium is of great interest today. Furthermore, the long-chain alcohol 1-undecanol (or undecanol) is liquid at $289.15 \mathrm{~K}$ but it is solid below this temperature. Around this temperature, different shapes of the equilibrium diagrams can be obtained, with regions in the presence of 
solid 1-undecanol. Changes in the temperature could vary the solid quantity, the composition of the equilibrium phases, for instance, and for these reasons, the influence of the temperature on the liquid-liquid, solid-liquid-liquid and solid-liquid equilibria of the water + ethanol +1 undecanol ternary system needs to be known.

The objective of this work has been to analyze the different regions in the water + ethanol +1 undecanol system appeared at different temperatures. Five different temperatures (275.15, 278.15, 281.15, 288.15 and 298.15 K) have been select to study the evolution of the liquidliquid, solid-liquid-liquid and solid-liquid regions formed. This kind of data has not been found in previously published articles.

\section{EXPERIMENTAL}

Ethanol and 1-undecanol were supplied by Merck, with a stated purity of 99.8 and 98 mass \% respectively. Water was purified with a Nanopure Barnstead ultrapure system. The experimental determination was carried out by preparing heterogeneous known mixtures of water, ethanol and 1-undecanol. This latter component was weighed in a tube and cooled at a lower temperature than its melting point. Then the solid was put in contact with cool mixtures of known concentration of water and ethanol. This methodology was applied in previous papers [1], [2] to prevent the formation of a gel that made the separation of phases difficult. The equilibrium between solid undecanol and the liquid water + ethanol mixture was reached in different thermostatic baths (275.15, 278.15, 281.15, 288.15 and $298.15 \mathrm{~K}$ ) where they were left until the equilibrium was reached and the different phases were separated clearly. Subsequently, the liquid phases were separated and prepared for analysis, with the addition of 2-propanol as an internal standard. Several set of samples were prepared and analyzed at different temperatures in order to 
ensure reproducibility of the tie lines and to determine the extension of the regions in the presence of 1-undecanol.

All the liquid samples were taken and analyzed by gas chromatography (Shimadzu GC-14A), equipped with a thermal conductivity detector (TCD), to determine the water and ethanol concentration. Injection volumes of the liquid samples were $1.0 \mu \mathrm{L}$. The separation of the components was carried out in a $2 \mathrm{~m} \times 3 \mathrm{~mm}$ column packed with Porapak Q 80/100. The column temperature was held at $453.15 \mathrm{~K}$, while the injector and detector temperatures were kept at $473.15 \mathrm{~K}$. The helium flow rate was $50 \mathrm{~mL} \cdot \mathrm{min}^{-1}$. Furthermore the water content in each organic phase was verified using the Karl Fisher technique. Water in the aqueous phase and 1undecanol in the organic phase was inferred by difference.

The small quantities of 1-undecanol in the aqueous phases were analyzed by gas chromatography using a Thermo Trace Fischer with a DB 624 column with a flame ionization detector (FID). The column temperature was held at $313.15 \mathrm{~K}(0.1 \mathrm{~min})$ and rose to $523 \mathrm{~K}$ by $40 \mathrm{~K} \mathrm{~min}^{-1}$ ramp rate and then the oven temperature was maintained for two minutes at $523 \mathrm{~K}$; the detector temperature was kept at $573.15 \mathrm{~K}$, while the injection port temperature was held at $523.15 \mathrm{~K}$; injections were performed on the split 1/50 mode, and Helium was used as a carrier at a rate of 1 $\mathrm{mL} \min ^{-1}$ after split. In this phase the water content was determined by difference.

The relative uncertainty of the mass fraction measurements was $2 \%$ for the minority components of each phase, calculated by the analysis of prepared standards. The experimental concentrations obtained and the known overall compositions of the heterogeneous mixture were used to check the mass balance, taking into account the accuracy of the concentration determination. A method of data reconciliation [6] was applied to check and fit liquid-liquid equilibrium data obtained 
experimentally, by slightly changing the analytical concentration, within the interval given by the uncertainties of the determination to satisfy the material balances. The results presented for these phases in the tables of the next section correspond to values obtained with this method.

\section{RESULTS}

Tables 1-5 present the experimental data obtained for each system. The phase diagrams for these systems are shown in Figures 1-5. As can be observed, the ternary systems at 275.15, 278.15 and 281.15 K present a region with three phases (S-L-L), a region with two phases (S-L) and a region of two liquid phases (L-L), which becomes greater as the temperature increases (Figures 1, 2 and 3). The diagrams at 288.15 and $298.15 \mathrm{~K}$ only present a heterogeneous region that contains two liquid phases (Figures 4 and 5). In this range the liquid-liquid equilibrium is not very sensitive to temperature changes. 
Table 1. Experimental Equilibrium Data for the system water (1) + ethanol (2) + 1-undecanol (3) for mass fractions $w_{i}$ at the Temperature $T=275.15 \mathrm{~K}^{\mathrm{a}}$.

\begin{tabular}{|c|c|c|c|c|c|}
\hline \multicolumn{3}{|c|}{ Aqueous phase } & \multicolumn{3}{|c|}{ Organic phase } \\
\hline$w_{1}$ & $w_{2}$ & $w_{3}$ & $w_{1}$ & $w_{2}$ & $w_{3}$ \\
\hline \multicolumn{6}{|c|}{ One liquid phase + one solid phase (1-undecanol) } \\
\hline 0.7013 & 0.2962 & 0.0025 & & & \\
\hline 0.7329 & 0.2640 & 0.0031 & & & \\
\hline 0.7793 & 0.2167 & 0.0040 & & & \\
\hline 0.8517 & 0.1432 & 0.0051 & & & \\
\hline 0.9196 & 0.0733 & 0.0071 & & & \\
\hline 0.9868 & 0.0016 & 0.0116 & & & \\
\hline \multicolumn{6}{|c|}{ Two liquid phases + one solid phase (1-undecanol) } \\
\hline 0.6592 & 0.3390 & 0.0018 & 0.0760 & 0.2010 & 0.7230 \\
\hline \multicolumn{6}{|c|}{ Two liquid phases } \\
\hline 0.5053 & 0.4737 & 0.0210 & 0.1828 & 0.3683 & 0.4489 \\
\hline 0.5684 & 0.4211 & 0.0106 & 0.1357 & 0.3190 & 0.5453 \\
\hline 0.6156 & 0.3828 & 0.0014 & 0.1024 & 0.2655 & 0.6321 \\
\hline 0.6514 & 0.3477 & 0.0016 & 0.0778 & 0.2111 & 0.7111 \\
\hline
\end{tabular}

$\square\left(\square_{L}\right) \square_{L}=0.02$ for the minority components of each phase. Water in the aqueous phase and 1undecanol in the organic phase have been determined by difference.

Table 2. Experimental (Liquid - Liquid) Equilibrium Data for the system water (1) + ethanol (2)

+ 1-undecanol (3) for mass fractions $w_{i}$ at the Temperature $T=278.15 \mathrm{~K}^{\mathrm{a}}$.

\begin{tabular}{|c|c|c|c|c|c|}
\hline \multicolumn{3}{|c|}{ Aqueous phase } & \multicolumn{3}{|c|}{ Organic phase } \\
\hline$w_{1}$ & $w_{2}$ & $w_{3}$ & $w_{1}$ & $w_{2}$ & $w_{3}$ \\
\hline \multicolumn{6}{|c|}{ One liquid phase + one solid phase (1-undecanol) } \\
\hline 0.7802 & 0.2170 & 0.0028 & & & \\
\hline 0.8501 & 0.1450 & 0.0049 & & & \\
\hline 0.9215 & 0.0717 & 0.0068 & & & \\
\hline 0.9896 & 0.0000 & 0.0104 & & & \\
\hline \multicolumn{6}{|c|}{ Two liquid phases + one solid phase (1-undecanol) } \\
\hline 0.7360 & 0.2620 & 0.0020 & 0.0600 & 0.1400 & 0.8000 \\
\hline \multicolumn{6}{|c|}{ Two liquid phases } \\
\hline 0.5546 & 0.4410 & 0.0044 & 0.1260 & 0.3720 & 0.5020 \\
\hline 0.5847 & 0.4130 & 0.0024 & 0.1110 & 0.3040 & 0.5850 \\
\hline 0.6428 & 0.3556 & 0.0016 & 0.0962 & 0.2466 & 0.6572 \\
\hline
\end{tabular}

$\square\left(\square_{D}\right) \square_{L}=0.02$ for the minority components of each phase. Water in the aqueous phase and 1undecanol in the organic phase have been determined by difference. 
Table 3. Experimental (Liquid - Liquid) Equilibrium Data for the system water (1) + ethanol (2) +1 -undecanol (3) for mass fractions $w_{i}$ at the Temperature $T=281.15 \mathrm{~K}^{\mathrm{a}}$.

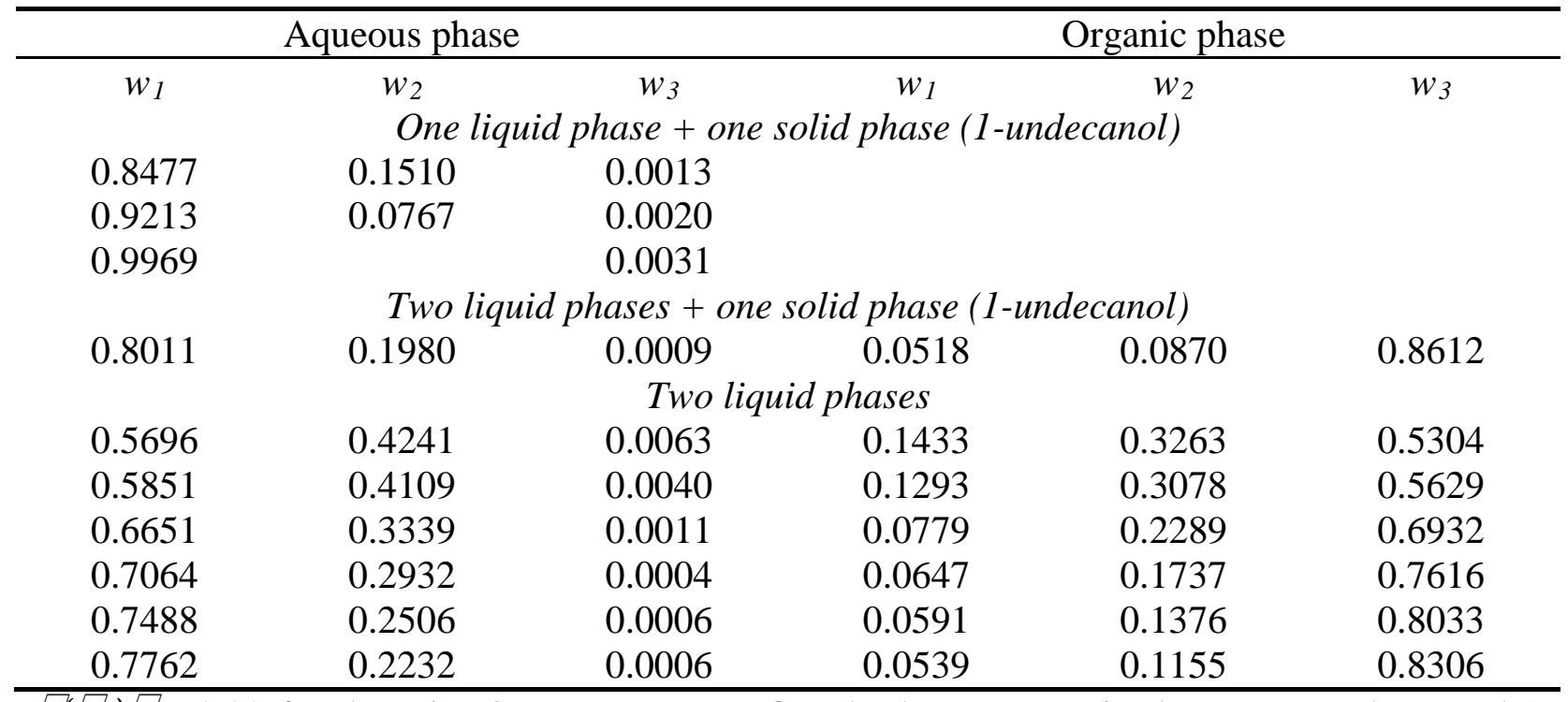

$\square\left(\square_{L}\right) L_{L}=0.02$ for the minority components of each phase. Water in the aqueous phase and 1undecanol in the organic phase have been determined by difference.

Table 4. Experimental (Liquid - Liquid) Equilibrium Data for the system water (1) + ethanol (2) + 1-undecanol (3) for mass fractions $w_{i}$ at the Temperature $T=288.15 \mathrm{~K}^{\mathrm{a}}$.

\begin{tabular}{cccccc}
\hline \multicolumn{3}{c}{ Aqueous phase } & \multicolumn{3}{c}{ Organic phase } \\
\hline$w_{1}$ & $w_{2}$ & $w_{3}$ & $w_{1}$ & $w_{2}$ & $w_{3}$ \\
0.5829 & 0.4106 & 0.0065 & 0.1748 & 0.3508 & 0.4744 \\
0.6013 & 0.3946 & 0.0041 & 0.1560 & 0.3253 & 0.5187 \\
0.6481 & 0.3502 & 0.0018 & 0.1077 & 0.2593 & 0.6330 \\
0.6994 & 0.3001 & 0.0005 & 0.0814 & 0.1954 & 0.7232 \\
0.7592 & 0.2406 & 0.0002 & 0.0598 & 0.1332 & 0.8070 \\
0.8210 & 0.1787 & 0.0003 & 0.0498 & 0.1014 & 0.8488 \\
0.8823 & 0.1172 & 0.0005 & 0.0419 & 0.0661 & 0.8920 \\
0.9990 & & 0.0010 & 0.0340 & & 0.9660 \\
\hline
\end{tabular}

$\square\left(\square_{L}\right) \square_{L=} 0.02$ for the minority components of each phase. Water in the aqueous phase and 1undecanol in the organic phase have been determined by difference. 
Table 5. Experimental (Liquid - Liquid) Equilibrium Data for the system water (1) + ethanol (2) +1 -undecanol (3) for mass fractions $w_{i}$ at the Temperature $T=298.15 \mathrm{~K}^{\mathrm{a}}$.

\begin{tabular}{cccccc}
\hline \multicolumn{3}{c}{ Aqueous phase } & \multicolumn{3}{c}{ Organic phase } \\
\hline$w_{1}$ & $w_{2}$ & $w_{3}$ & $w_{1}$ & $w_{2}$ & $w_{3}$ \\
0.5974 & 0.3949 & 0.0077 & 0.1517 & 0.3271 & 0.5212 \\
0.6482 & 0.3494 & 0.0024 & 0.0995 & 0.2598 & 0.6407 \\
0.7039 & 0.2934 & 0.0028 & 0.0728 & 0.1989 & 0.7283 \\
0.8143 & 0.1827 & 0.0030 & 0.0396 & 0.0911 & 0.8693 \\
0.8734 & 0.1234 & 0.0032 & 0.0416 & 0.0574 & 0.9010 \\
\hline
\end{tabular}

$\square\left(\square_{L} L_{L}=0.02\right.$ for the minority components of each phase. Water in the aqueous phase and 1undecanol in the organic phase have been determined by difference. 


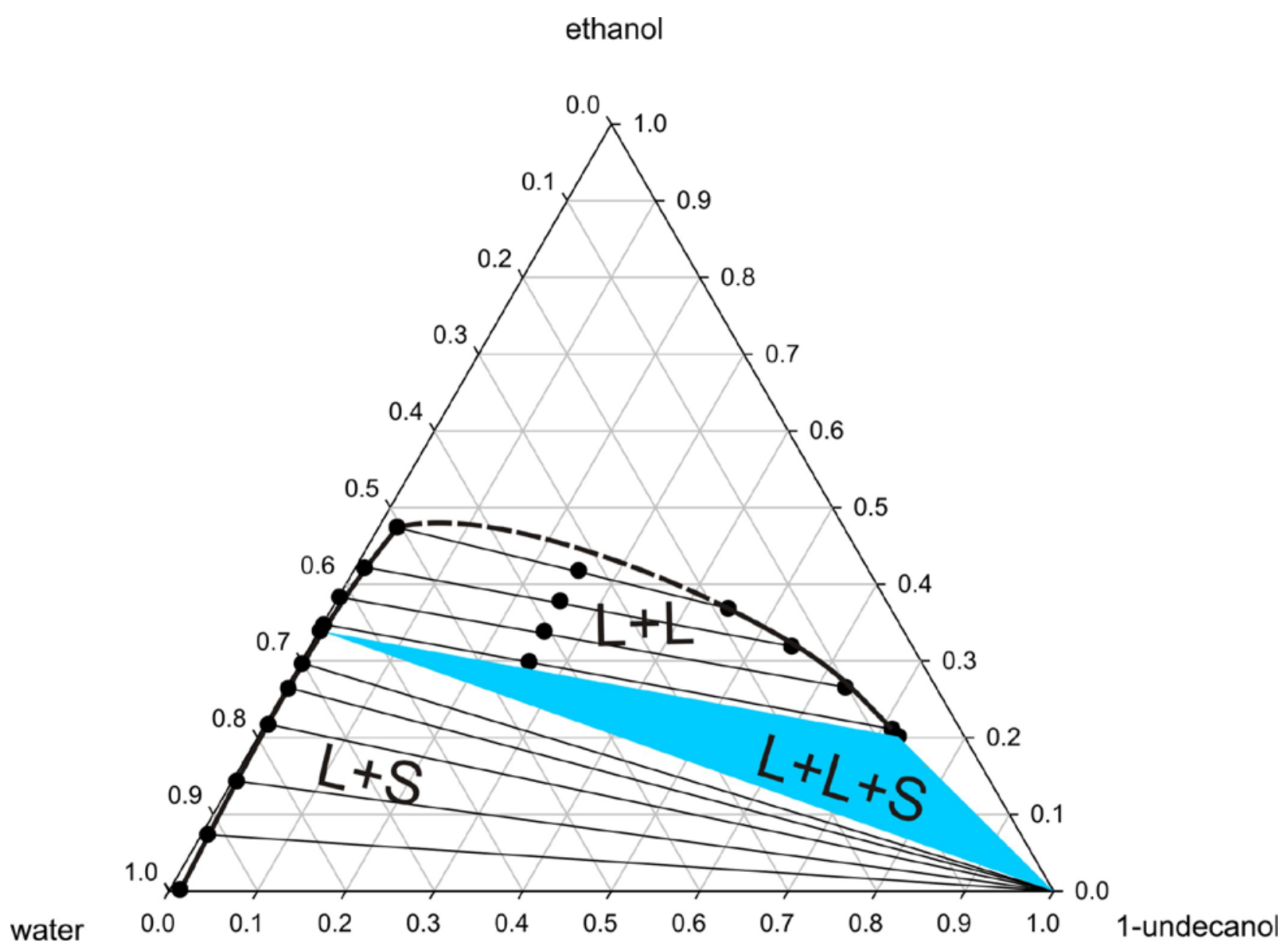

Figure 1. Solid-liquid-liquid and liquid-liquid equilibria (mass fraction) for the ternary system water + ethanol +1 -undecanol at the Temperature $T=275.15 \mathrm{~K}$. 


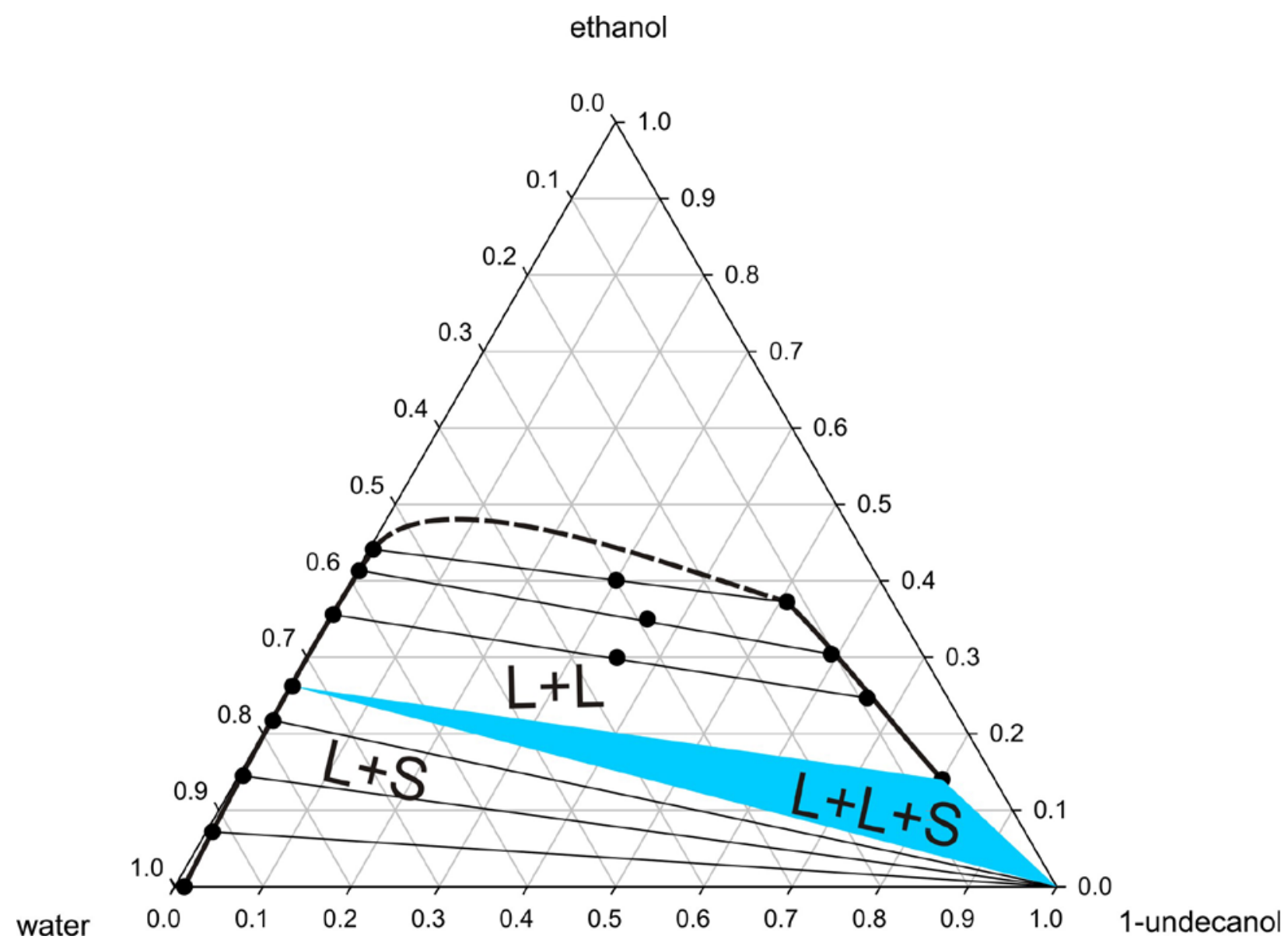

Figure 2. Solid-liquid-liquid and liquid-liquid equilibria (mass fraction) for the ternary system water + ethanol +1 -undecanol at the Temperature $T=278.15 \mathrm{~K}$. 


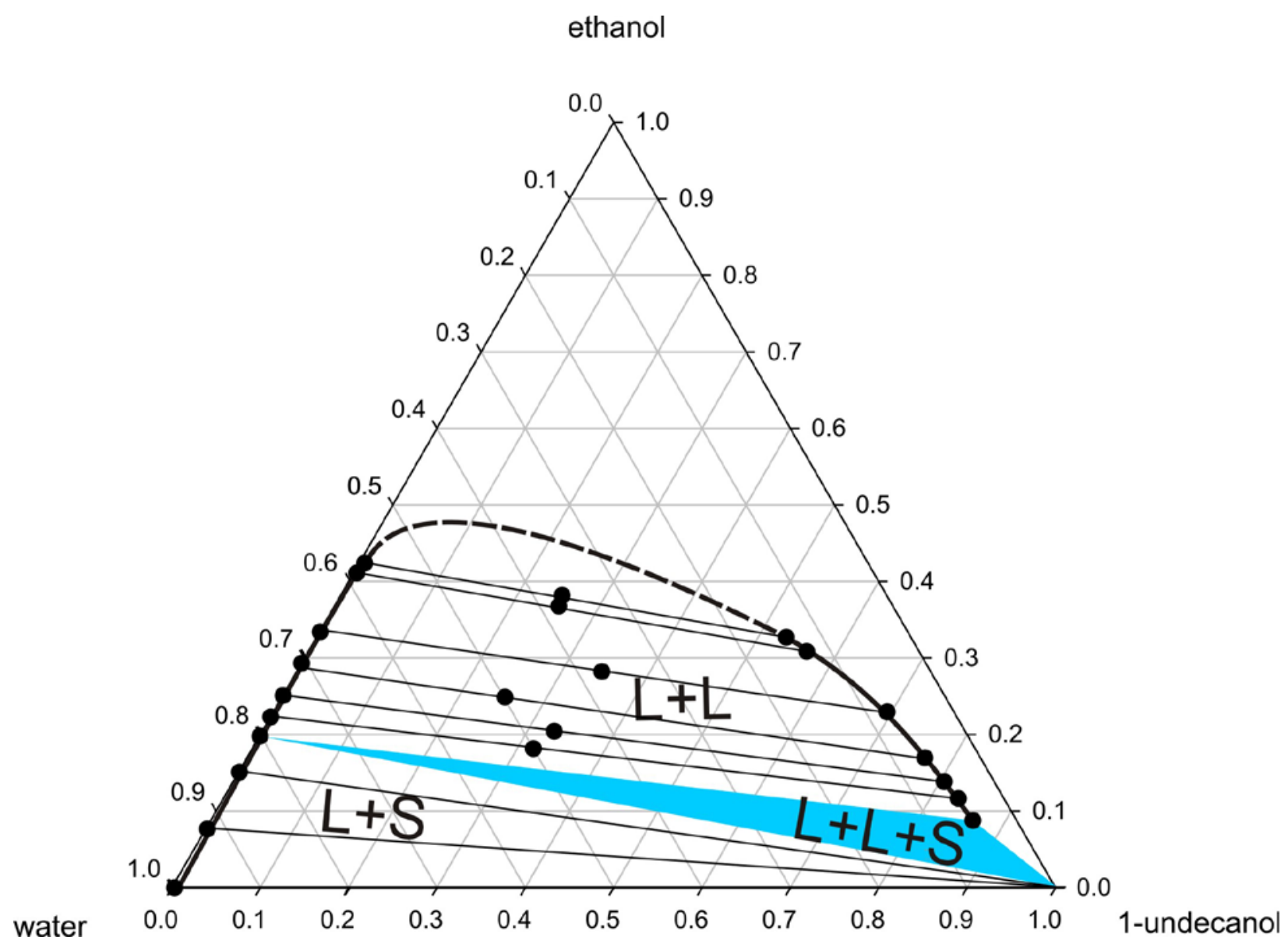

Figure 3. Solid-liquid-liquid and liquid-liquid equilibria (mass fraction) for the ternary system water + ethanol +1 -undecanol at the Temperature $T=281.15 \mathrm{~K}$. 


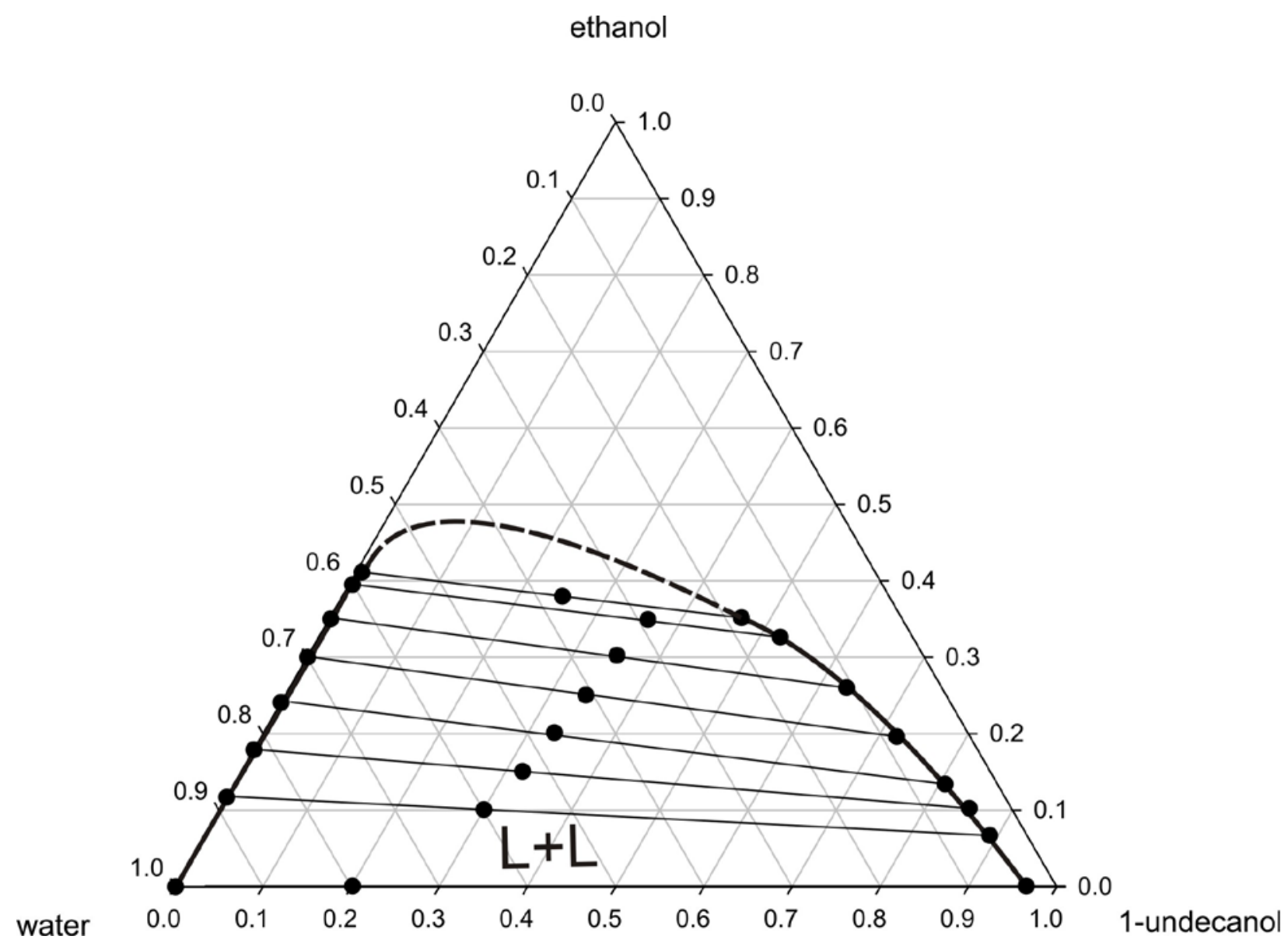

Figure 4. Liquid-liquid equilibria (mass fraction) for the ternary system water + ethanol +1 undecanol at the Temperature $T=288.15 \mathrm{~K}$. 


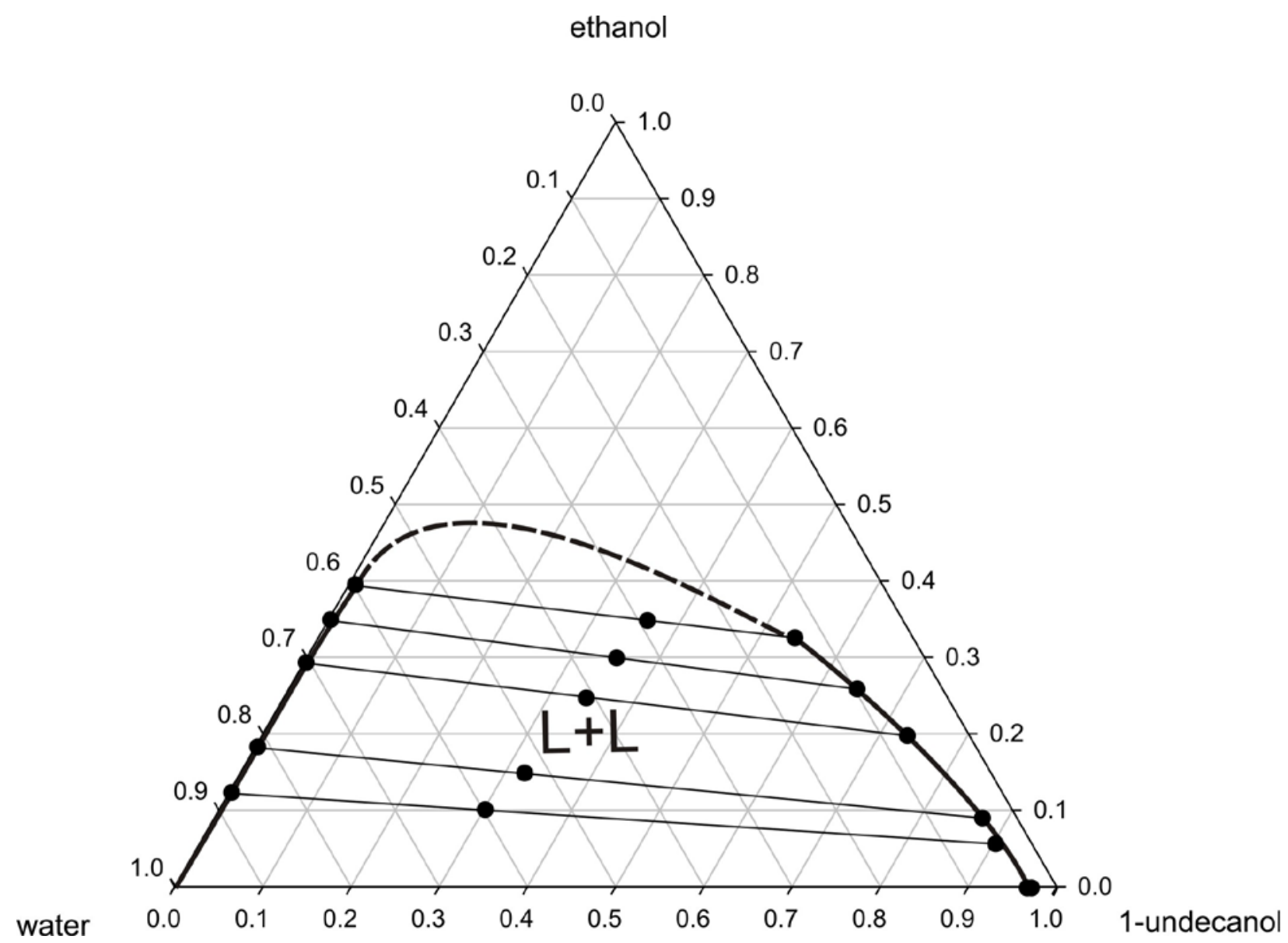

Figure 5. Liquid-liquid equilibria (mass fraction) for the ternary system water + ethanol +1 undecanol at the Temperature $T=298.15 \mathrm{~K}$. 
With respect to experimental data found in the scientific literature, Góral et al. [7] reported results of alkanol + water systems, comprising of 1-undecanol + water between 288.15 and 363.65 K. In their paper they include data from Tokunaga et al. [8] of the mutual solubility of water and 1-undecanol at $288.15 \mathrm{~K}$ (3.46 mass \% of water in 1-undecanol) and at $298.15 \mathrm{~K}$ (3.44 mass \% of water in 1-undecanol). On the other hand, Gündogdu and Çehreli showed ternary liquid-liquid phases equilibria of water +1 -undecanol with several carboxylic acids [9] and consider at $298.15 \mathrm{~K}$ the water solubility in 1-undecanol as 2.98 mass \%, similar to the previous data and in accordance with our experimental results.

In previous work [1-2], ternary phase equilibria was obtained at different temperatures for other heavy alcohols (1-decanol and 1-dodecanol). Taking into account these results the following conclusions can be drawn:

a) For temperatures above $298.15 \mathrm{~K}$, all the diagrams show a similar shape, there is only a two-liquid region and the width of the heterogeneous region is quite similar.

b) At low temperatures, for example $275.15 \mathrm{~K}$, the three ternary systems present different regions containing solid.

c) The temperature at which the solid phase appears depends on the long-chain alcohol studied. For 1-decanol this temperature is $275.15 \mathrm{~K}$, for 1-undecanol is between 281.15 and $285.15 \mathrm{~K}$ and in the case of 1-dodecanol is $296.15 \mathrm{~K}$.

d) Comparing the three ternary diagrams at the same temperature, for example $288.15 \mathrm{~K}$ (Figure 6), can be observed the similar width of the heterogeneous region. It is worth to point out that a solid phase appears in the ternary diagram of the alcohol with the greatest melting point (1-dodecanol), while the other two diagrams only have a two-liquid region. 

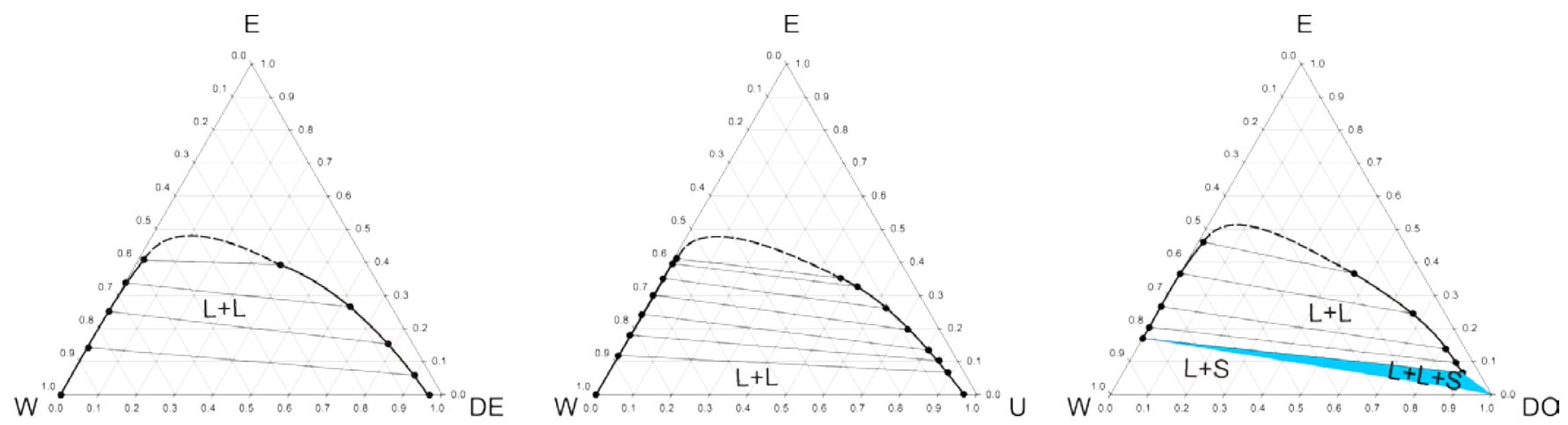

Figure 6. Liquid-liquid equilibria (mass fraction) for the ternary systems water (W) + ethanol $(E)+1$-undecanol (U), water (W) + ethanol (E)+ 1-decanol (DE), water (W) + ethanol (E)+ 1dodecanol (DO) at the temperature $T=288.15 \mathrm{~K}$.

The changes observed in the shape of the equilibrium diagram can be used to improve the process of extraction of ethanol from aqueous solutions using 1-undecanol as a solvent or to investigate new analytical techniques, such as DLLME-SFO, with the use of extraction solvents with lower toxicity in environmentally sustainable processes.

\section{CONCLUSIONS}

Different shapes of the equilibrium diagrams have been observed for the water - ethanol - 1undecanol ternary systems, which have been determined experimentally at different temperatures and atmospheric pressure. Only a L-L region is observed at 288.15 and $298.15 \mathrm{~K}$, which is not very sensitive to temperature changes. When the temperature decreases, three regions with different areas are present at 275.15, 278.15 and $281.15 \mathrm{~K}$ : two with solid presence (S-L-L and S-L) and a region of two liquid phases (L-L), which becomes greater as the temperature increases. The changes observed in the shape of the equilibrium diagram can be used to improve the process of extraction of ethanol from aqueous solutions using 1-undecanol as a solvent or to 
investigate new analytical techniques using extraction solvents with lower toxicity in environmentally sustainable processes.

\section{ACKNOWLEDGMENT}

The authors wish to thank the University of Alicante (Spain) for the financial support.

\section{ABBREVIATIONS}

DLLME, dispersive liquid-liquid microextraction; DLLME-SFO dispersive liquid-liquid microextraction based on solidification of floating organic drop

\section{REFERENCES}

(1) Boluda, N.; Gomis, V.; Ruiz, F.; Bailador, H. The influence of temperature on the liquidliquid-solid equilibria of the ternary system water + ethanol + 1-dodecanol. Fluid Phase Equilib. 2005, 235, 99-103.

(2) Boluda, N.; Gomis, V.; Bailador, H.; Pequenin, A. Influence of the temperature on the liquidliquid-solid equilibria of the ternary system water + ethanol + 1-decanol. J. Chem. Eng. Data. 2011, 56, 65-68.

(3) Burdock, G.A. Encyclopedia of Food and Color Additives, Vol. I-III. $1^{\text {st }}$ ed. CRC Press, Boca Raton. 1997.

(4) Kocúrová, L.; Balogh, I. S.; Šandrejová, J.; Andruch, V. Recent advances in dispersive liquid-liquid microextraction using organic solvents lighter than water. A review. Microchem. J. 2012, 102, 11-17.

(5) Mirzaei, M.; Behzadia, M.; Abadia, N. M.; Beizaeia, A. Simultaneous separation/ preconcentration of ultra trace heavy metals in industrial wastewaters by dispersive liquid-liquid microextraction based on solidification of floating organic drop prior to determination by graphite furnace atomic absorption spectrometry. J. Hazard. Mat. 2011, 186, 1739-1743.

(6) Gomis, V.; Ruiz, F.; Asensi, J.C.; Saquete, M.D. Procedure for checking and fitting experimental liquid-liquid equilibrium data. Fluid Phase Equilib. 1997, 129, 15-19.

(7) Góral, M.; Wisniewska-Goclowska, B.; Maczynski, A. Recommended Liquid-Liquid Equilibrium Data. Part 4. 1-Alkanol-Water Systems J. Phys. Chem. Ref. Data. 2006, 35, 13911414.

(8) Tokunaga, S.; Manabe, M.; Koda, M. Mem. Niihama Technical College. Sci. Eng. 1980. 16, 96.

(9) Gündogdu, S.; Çehreli, S. Ternary liquid-liquid phase equilibria of (water-carboxylic acid1-undecanol) systems at 298.15 K. 2012. Fluid Phase Equilib. 2012, 331, 26-32. 